\title{
Lumen Border Detection of Intravascular Ultrasound via Denoising of Directional Wavelet Representations
}

\author{
Amin Katouzian $^{3}$, Elsa Angelini ${ }^{1}$, Auranuch Lorsakul ${ }^{3}$, Bernhard Sturm ${ }^{2}$, and \\ Andrew Laine ${ }^{3,4}$ \\ ${ }^{1}$ Institut TELECOM, TELECOM ParisTech, CNRS LTCI, Paris, France \\ ${ }^{2}$ Volcano Corporation, Rancho Cordova, CA USA \\ ${ }^{3}$ Biomedical Engineering and ${ }^{4}$ Radiology Departments, Columbia University, New York, NY USA \\ \{Laine@columbia.edu\}
}

\begin{abstract}
In this paper, intravascular ultrasound (IVUS) grayscale images, acquired with a single-element mechanically rotating transducer, are processed with wavelet denoising and region-based segmentation to extract various layers of lumen contours and plaques. First, IVUS volumetric data is expanded on complex exponential wavelet-like basis functions, also known as Brushlets, which are well localized in time and frequency domains. Brushlets denoising have demonstrated in the past a great aptitude for denoising ultrasound data and removal of blood speckles. A region-based segmentation framework is then applied for detection of lumen border layers, which remains one of the most challenging problems in IVUS image analysis for images acquired with a single element, mechanically rotating $45 \mathrm{MHz}$ transducer. We evaluated hard thresholding for Brushlet denoising, and compared segmentation results to manually traced lumen borders. We observed good agreement and suggest that the proposed algorithm has a great potential to be used as a reliable pre-processing step for accurate lumen border detection.
\end{abstract}

Key words: Brushlet, Intravascular Ultrasound (IVUS), Denoising, Border Detection, Lumen, Thresholding.

\section{Introduction}

Cardiovascular disease (CVD) remains the leading cause of death worldwide. In 2004, CVD took about 800,000 lives in the United States with more than half of them due to atherosclerotic plaques [1]. There has been extensive interest in developing new imaging modalities to screen the progression and regression of atherosclerotic plaques in the past decade. Among them, intravascular ultrasound (IVUS) is the most widely used, real-time, and inexpensive imaging modality that not only provides pathological as well as 


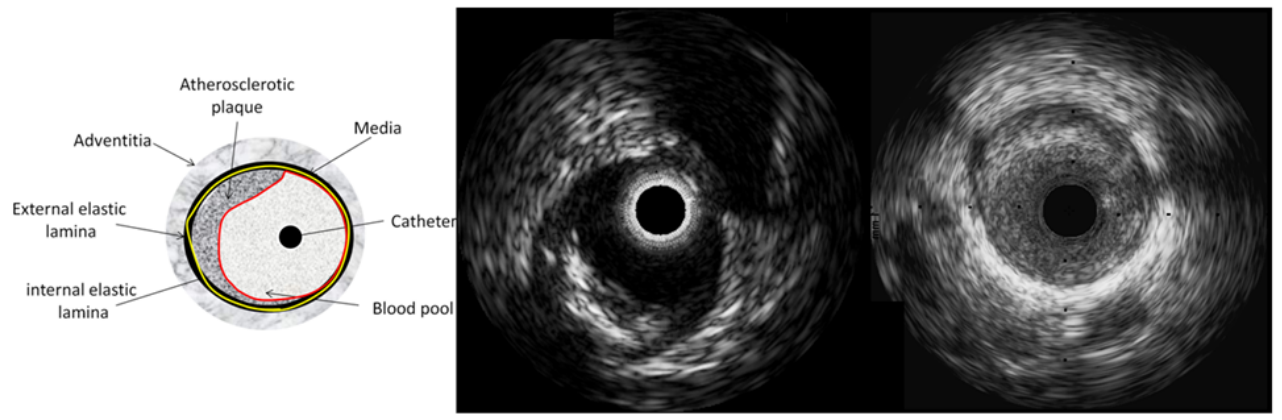

(a)

(b)

(c)

Fig. 1. Schematic cross-sectional anatomy of arterial wall and atherosclerotic plaque, the lumen border (red) and vessel wall border (yellow) (a), two distinct cross-sectional grayscale IVUS images acquired with 64elements phased-array $20 \mathrm{MHz}$ (b) and single-element rotating $45 \mathrm{MHz}$ (c) VOLCANO transducers.

morphological cross-sectional grayscale images of arterial walls but also makes atherosclerotic tissue characterization feasible, using IVUS radiofrequency (RF) signals and images [2,3]. IVUS image segmentation has been a subject of interest for researchers due to the rapidly growing use of this imaging modality in catheterization procedures for the following two main reasons. First, one important parameter during angioplasty or stent implantation procedure, is the ratio of lumen to vessel wall cross sectional area for stenosis regions. For instance, the interventional cardiologist uses these measurements to select the appropriate type, length and diameter of a stent. Secondly, vessel walls and lumen contours are required to be traced prior to tissue characterization and plaque RF signals extraction. Pullback IVUS data files contain thousands of cross-sectional images, automatic extraction of vessel wall and lumen borders has been the topic of only a few research efforts $[4,5,6,7]$ and remains a challenging image processing problem.

Clinical application of automated segmentation methods has seen limited success due to the presence of guide wire, the presence of arc of calcified plaques, the motion of the catheter as well as heart, and appearance of sub branches. Generally speaking, on IVUS image data, detection of the external vessel wall border is relatively easier than internal lumen border. Comparing IVUS ultrasound probes, the lumen border is better depicted in images acquired with a 64-element phased-array $20 \mathrm{MHz}$ transducer in comparison with those acquired with a single element mechanically rotating $45 \mathrm{MHz}$ transducer. At higher center frequency spatial resolution is improved, at the cost of more scattering from red blood cells inside the lumen. Figure 1 illustrates the schematic cross-sectional anatomy of arterial wall and atherosclerotic plaque along with two distinct cross-sectional grayscale IVUS images acquired with 64-elements phased-array $20 \mathrm{MHz}$ as well as a single-element rotating $45 \mathrm{MHz}$ VOLCANO transducer. 


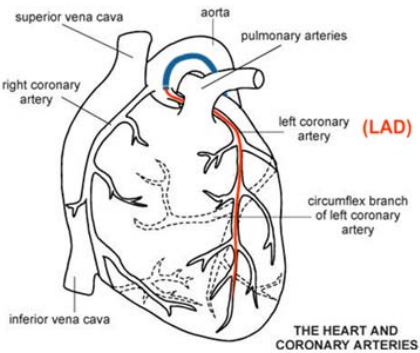

(a)

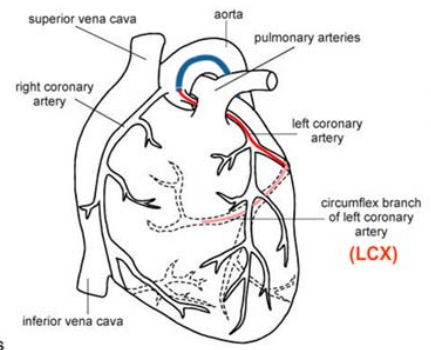

(b)

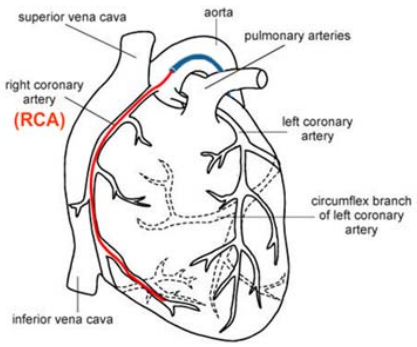

(c)

Fig. 2. The heart and coronary arteries. Schematic illustration of advanced catheter (red) into LAD (a) LCX (b) and RCA (c)

To improve image quality and be able to rely on a simple segmentation framework, we developped a three-dimensional (3D) Brushlet-based algorithm, taking into account blood flow during the pullback. The appearance of regions corresponding to plaques, vessel wall and surrounding fat is more stable within successive frames than for the blood pool. Based on this observation, expansion using a brushlet basis and thresholding operator was used to characterize persistent textures along the pull back dimension to remove random scattering blood and speckle. Denoising was performed in polar representation and results displayed in traditional Cartesian coordinates.

Segmentation of denoised images was performed with region-based optimal partitioning, combined with Markovian regularization, an iterated conditional mode (ICM) classification algorithm [8] was used, based on 4 classes. Extraction of the different layers, corresponding to the different ICM-classes, was finalized with a parametric deformable model.

This paper is organized as follows. Section 2 describes the acquisition system specification and data collection methodology. We review the Brushlet analysis, denoising and segmentation algorithms in Section 3. The experimental results are then demonstrated in Section 4. The paper is summarized with conclusions in Section 5.

\section{Data Collection}

We collected IVUS grayscale images from three patients using a single element mechanically rotating $45 \mathrm{MHz}$ Revolution ${ }^{\mathrm{TM}}$ transducer and $\mathrm{s5}^{\mathrm{TM}}$ imaging system manufactured by VOLCANO (Rancho Cordova, CA). The catheter was advanced on top of the guide wire from the femoral artery toward the site of coronary arterial occlusion (i.e. right coronary artery (RCA), left anterior descending (LAD), left circumflex (LCX)) via aorta. Figure 2 illustrates schematically the heart, coronary arteries and the catheter 


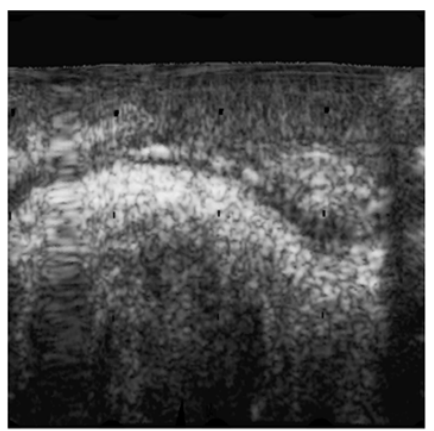

(a)

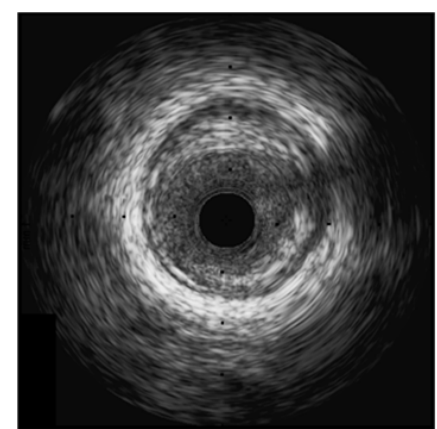

(b)

Fig. 3. Grayscale IVUS image in polar (a) and Cartesian (b) domains

path. During image acquisition, the catheter is pulled back from the distal to proximal locations with a speed of $0.5 \mathrm{~mm} / \mathrm{sec}$, acquiring 30 frames $/ \mathrm{sec}$. Ultimately, each grayscale IVUS frame was constructed consisting of 500 radial lines that span $360^{\circ}$ and 500 samples/line after decimation and interpolation in radial and lateral directions, respectively. The original images, acquired in polar coordinates, were mapped to Cartesian coordinates to construct typical IVUS images, as shown in Figure 3.

\section{Methodology}

We apply a 3D (cross-sectional image of arterial content + pull-back distance) overcomplete brushlet analysis to sub-blocks of IVUS frames in polar coordinates during each pullback. We assume that there is a strong spatial coherence in the appearance of plaque, arterial wall and surrounding fats within blocks of the analysis. This coherence is organized in concentric circular layers and is better expressed and visualized in polar representations of the image data. Also, brushlet tiling and expansion is based on a regular square lattice which is better correlated with homogeneous textured patterns in polar $(r, \theta)$ rather than Cartesian $(x, y)$ image representation. The presence of blood speckles within the volume of analysis is also considered to be dynamic due to blood flow. The underlying hypothesis for bruhlet denoising via thresholding, is that blood speckle can be removed by including the pull-back dimension in the textural brushlet analysis in the frequency domain. Finally, the results are mapped back to Cartesian coordinates for visual display. 


\subsection{Brushlet Basis}

Brushlets are windowed complex exponential functions in the family of steerable wavelets and were introduced in [9]. They divide the real axis into subintervals $\left[a_{n}, a_{n+1}\right]$ of length $l_{n}$, and define a brushlet analysis function as follows

$u_{j, n}(x)=b_{n}\left(x-l_{n} / 2\right) e_{j, n}(x)+v\left(x-a_{n}\right) e_{j, n}\left(2 a_{n}-x\right)-v\left(x-a_{n+1}\right) e_{j, n}\left(2 a_{n+1}-x\right)$

The $b_{n}$ and $v$ are two windowed functions, which are defined from the ramp function. The complex orthonormal basis function $u_{j, n}$, can be constructed using these two functions along with the complex exponential function $e_{j, n}$ that is defined as $e_{j, n}(x)=\frac{1}{\sqrt{l_{n}}} e^{-2 i \pi j \frac{\left(x-a_{n}\right)}{l_{n}}}$. Given any one-dimensional signal $f$ in $L^{2}(R)$, its Fourier

transform $\hat{f}$ can be projected onto the brushlet basis as $\hat{f}=\sum_{n} \sum_{j} \hat{f}_{n, j} u_{n, j}$ where $\hat{f}_{n, j}$ are the brushlet coefficients. It has been shown that the projected of $\hat{f}$ onto the brushlet basis can be implemented in an efficient fashion using a folding technique and fast Fourier transform (FFT) [10]. In the reconstruction process, the original signal can be retrieved using inverse Fourier transform and unfolding operation.

In our application, a tensor product extension was used for the volume of IVUS data frames. Consequently, such an extension would lead to the orientation selectivity in the brushlet domain, which is described next.

\subsection{Frequency Tilling and Overcomplete Representation}

The decomposition of IVUS volumes onto the brushlet orthonormal basis provides selectable textural features with different orientations in the Fourier (brushlet) domain. Since we deal with volumetric datasets, this can be reliably performed by tilling the Fourier domain into quadrants (sub-cubes), each representing a specific size and orientation of a brushstroke, which is analogous to a wavelet scaling function.

We used an overcomplete multiscale representation of the brushlet coefficients for two reasons. First, we avoided aliasing effect and secondly, we intended to preserve as much textural information as possible in the transform domain due to the stringent behavior of blood and plaque signals that makes the lumen border detection very challenging. 


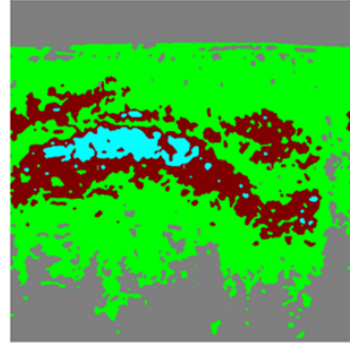

(a)

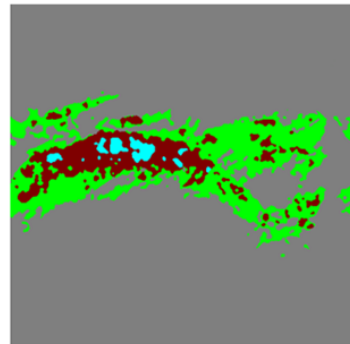

(e)

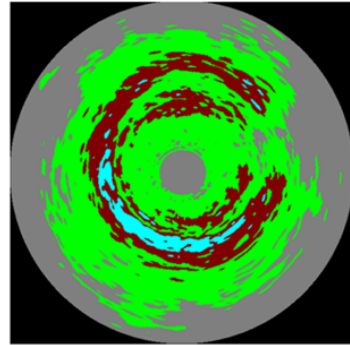

(b)

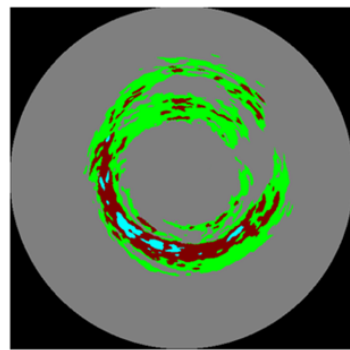

(f)

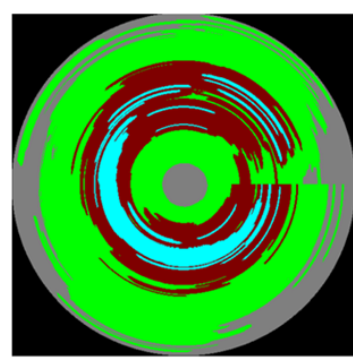

(c)

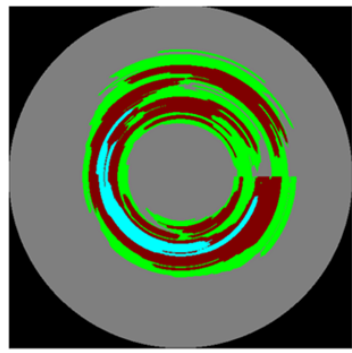

(g)

Fig. 4. ICM segmentation. 4-classes ICM results for original image in polar (a), Cartesian coordinates (b) and after dilation (c). 4-classes ICM results for denoised image in polar (d), Cartesian coordinates (e) and after dilation (f).

\subsection{Thresholding for Removal of Blood Speckle}

The purpose of this study is to offer a reliable denoising algorithm by removing blood speckle (noise), such that the resulting denoised data can be employed as an input for any 2D or 3D segmentation algorithm. We used two methods for eliminating blood noise via thresholding brushlet coefficients. We deployed a hard thresholding technique on both magnitude and real part of brushlet coefficients.

\subsection{Segmentation of IVUS Data}

We used the iterative conditional model (ICM) segmentation framework to segment the IVUS data into 4 regions (layers), characterized by their average gray values. The ICM segmentation framework corresponds to a discrete minimization of the piece-wise Mumford and Shah functional energy [11] replacing curvature constraints with a Markovian regularization of the contours. The Markovian regularization provides a very efficient computational framework to control orthogonal and vertical associations of 


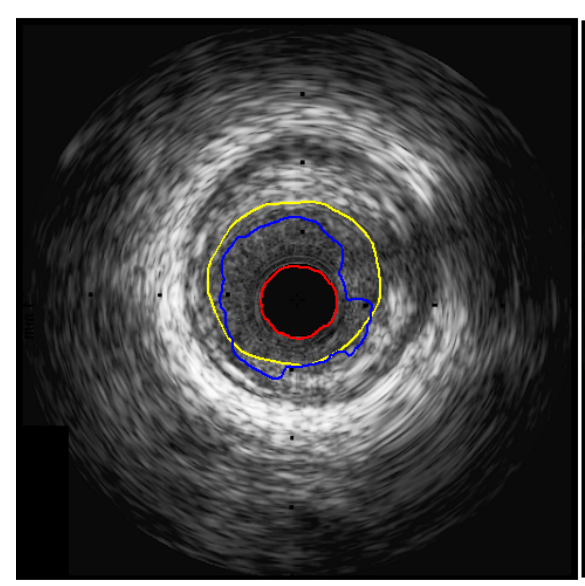

(a)

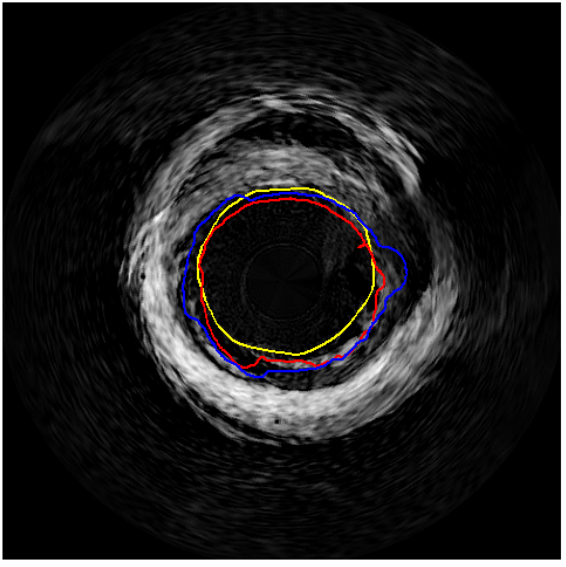

(b)

Fig. 5. Segmentation results at different layers (red, Blue) along with manually traced contour by an expert (yellow) on original (a) and denoised (b) IVUS images.

similar class labels when applied on a regular lattice corresponding to 4-neighbor pixels in 2D. Such a constraint is ideal for circular patterns such as vessel layers, when displayed in polar coordinates. We therefore applied the ICM on the IVUS original and denoised volumes in polar coordinates. Consequently, classification results were transformed to the Cartesian coordinates for visualization and extraction of the center contours with a standard parametric deformable model applied on thresholded ICM classifications for the four classes and imposing a regular growth of vessel contour areas by iteratively initializing the deformable model at the center of the transducer. This extraction step was performed after dilation of the ICM classification results with a horizontal line structuring element of 70 pixels, in polar coordinates. This computational step was designed to close the open and unconnected borders to obtain better results from parametric deformable model and estimate the lumen border more precisely, as illustrated in Figure 4.

\section{Results AND Discussion}

In the first experiment, we processed IVUS volumes of size $512 \times 512 \times 2$ and the Fourier domain was tilled using four, four and two cubes in $\mathrm{x}, \mathrm{y}$ and pull-back dimension respectively each with overcomplete representation. In this case, the brushstroke orientation was $\pm 90, \pm 90$ and \pm 180 in $x, y$ and pull-back direction, respectively. We 
repeated the experiment using volumes of size $512 \times 512 \times 8$ to insure adequate spatial resolution in the pull-back dimension (8 slice depth). Higher spatial resolution in this dimension resulted in blurred denoised data due to the effects of the motion of the catheter and heart.

Figure 5 illustrates the results of our segmentation algorithm, in the second experiment, on original IVUS image and resulting denoised image using a hard thresholding operator. For this purpose, the thresholding was performed in each cube empirically for the threshold value of $50 \%$ of the maximum value of real part of the coefficients. The resulting denoised coefficients were then classified into four groups with uniform gray level values for ICM initialization. However, we only extracted the contours from the first two layers since they better approximated the true lumen border. As we can see, the segmentation algorithm outperforms on denoised images and the automated borders at two layers (red L1 and blue L2) are well correlated and matched with manually traced lumen border (yellow) traced by an expert cardiologist.

For both experiments the regression analysis was performed to evaluate the agreement between automated and manual traced contours for 104 frames within a pullback, Figure 6(a). In addition, we computed the Tanimoto coefficients to see how well the automated and manual contours are matched throughout the pullback, Figure 6(b). The average Tanimoto coefficients for L1 and L2 borders in the first experiment, using block size of $512 \times 512 \times 2$, found to be $41.75 \pm 13.66$ and $69.16 \pm 7.37$, respectively, which was less than the computed Tanimoto coefficients for the second experiment using block size of $512 \times 512 \times 8$ IVUS frames (case \#1, Table 1 ). The Tanimoto coefficients show the highest agreement in positioning of automated and manually traced borders. According to Figure 6(b), the higher Tanimoto coefficients were achieved for the first 50 frames where the pullback was more consistent. Our results show that the non-coherent pattern of blood speckle is better captured in longer IVUS volumes although the changes in plaque textures or motion artifacts may be more observed that can degrade the results (frames 51 and 81 in figure 6(a)). This suggests an adaptive processing technique in future. Table 1 summarizes the Tanimoto coefficients statistics as well as correlation values for 233 frames extracted from three distinct cases using block size of $512 \times 512 \times 8$ IVUS frames. Overall, the extracted contour at the second layer (L2) found to have more agreement with manually traced borders.

In the third experiment, we processed the IVUS data with the same parameters whereas the hard thresholding technique was applied on the magnitude of brushlet coefficiets in each cube with threshold value of $15 \%$ of the maximum value. Movie 1, demonstrates the sequence of original (left hand side) and denoised (right hand side) images through the pullback. In this video, a counter-clock-wise vortex type patterns is visible. Regardless of 


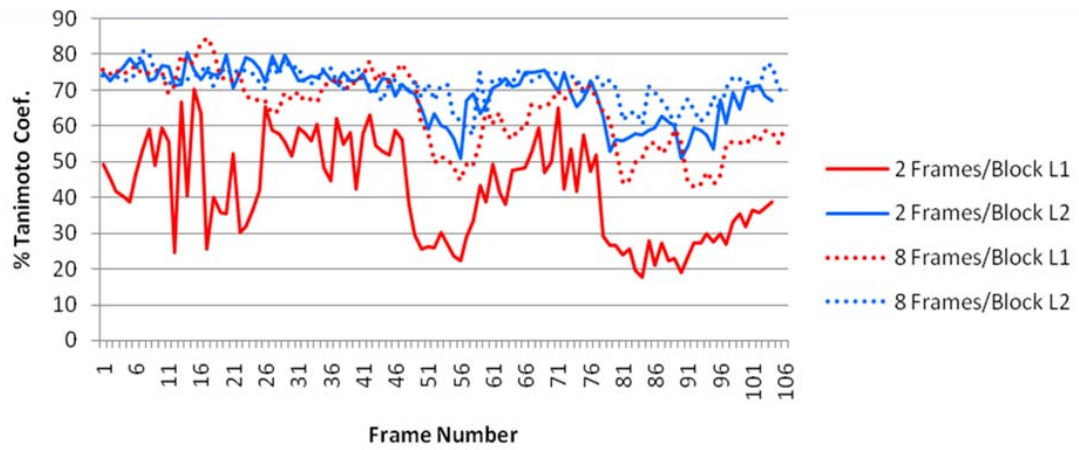

(a)

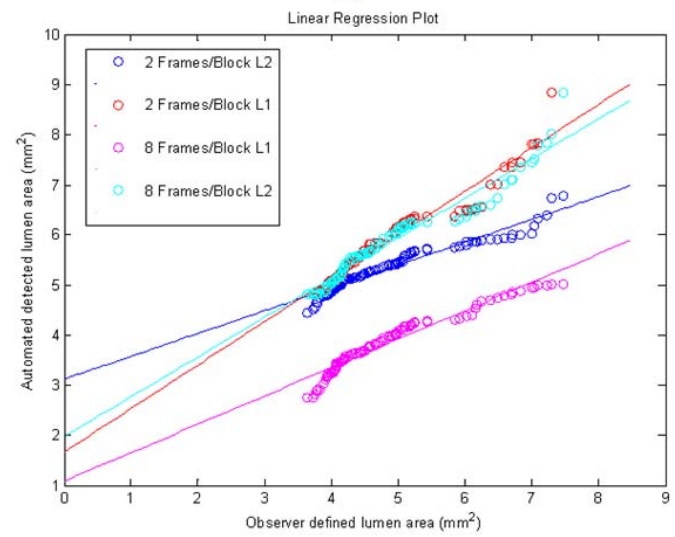

(b)

Fig. 6. Evaluation of detected contours at two layers (L1, L2) in 104 IVUS frames within a pullback with two different block sizes. Tanimoto coefficients (a), Regression analysis (b).

source of such coherent dynamic patterns, the results become useful, particularly for images acquired with $45 \mathrm{MHz}$ transducers, when the expert tries to delineate the lumen border visually. Genrally, experts go back and forth among frames in order to locate the lumen border visually and draw it manully. Our results not only ease this process but also make other techniques attractive such as optical flow to track these vortices and estimate the lumen border.

\section{Conclusion}

In this paper we presented a new 3D denoising technique based on brushlet representations to remove blood noise in IVUS images acquired with a $45 \mathrm{MHz}$ single- 
Table 1. Tanimoto coefficient statistics and correlation between automated and manually traced borders in 233 frames collected from three distinct cases.

\begin{tabular}{|l|l|l|l|l|l|}
\cline { 2 - 6 } \multicolumn{1}{c|}{} & $\begin{array}{c}\text { Number } \\
\text { of Frames }\end{array}$ & $\begin{array}{c}\text { Mean } \\
\text { Tanimoto } \\
\text { Coefficients }\end{array}$ & $\begin{array}{c}\text { Maximum } \\
\text { Tanimoto } \\
\text { Coefficients }\end{array}$ & $\begin{array}{c}\text { Minimum } \\
\text { Tanimoto } \\
\text { Coefficients }\end{array}$ & $\begin{array}{c}\text { Correlation } \\
(\boldsymbol{p}<\mathbf{0 . 0 0 0 1 )}\end{array}$ \\
\hline Case \# 1 & 104 & $L 1: 64.12 \pm 10.56$ & $L 1: 84.55$ & $L 1: 42.78$ & $L 1: 79.01$ \\
& & $L 2: 71.34 \pm 4.74$ & $L 2: 81.02$ & $L 2: 57.53$ & $L 2: 78.74$ \\
\hline Case \# 2 & 104 & $L 1: 71.10 \pm 9.69$ & $L 1: 84.20$ & $L 1: 48.33$ & $L 1: 0.78$ \\
& & $L 2: 72.70 \pm 4.05$ & $L 2: 81.09$ & $L 2: 60.87$ & $L 2: 0.80$ \\
\hline Case \# 3 & 25 & $L 1: 69.73 \pm 4.22$ & $L 1: 77.69$ & $L 1: 60.47$ & $L 1: 0.94$ \\
& & $L 2: 77.08 \pm 4.63$ & $L 2: 86.93$ & $L 2: 70.14$ & $L 2: 0.95$ \\
\hline
\end{tabular}

element transducer. We further used denoised volumes as input to a 3D multi-region multi-channel segmentation algorithm to estimate the lumen border. We observed that the algorithm performs fairly well especially in consistent pullback where there is a strong spatial coherence in the appearance of plaque. In future, we will try to make the thresholding adaptively and look for more informative directions that provide more distinct differentiation between blood and non-blood textures.

\section{References}

[1] American Heart Association, Heart Disease and Stroke Statistics - 2006 update, American Heart Association 2006.

[2] A. Nair, B. D. Kuban, N. Obuchowski, D. G. Vince, "Assessing spectral algorithms to predict atherosclerotic plaque composition with normalized and raw intravascular ultrasound data. Ultrasound in Med. \& Biol., vol. 27, no. 10, pp. 1319-1331, 2001.

[3] A. Katouzian, B.Baseri, E. E. Konofagou, A. F. Laine, "An alternative Approach to Spectrum-Based Atherosclerotic Plaque Characterization Techniques Using Intravascular Ultrasound (IVUS) Backscattered Signals,” MICCAI-CVII 2008.

[4] M. H. R. Cardinal, J. Meunier, G. Soulez, R. L. Maurice, E. Therasse, G. Cloutier, "Intravascular Ultrasound Image Segmentation: A Three-Dimensional Fast-Marching Method Based on Gray Level Distribution, “ IEEE Tran. Med. Imag., vol. 25, no. 5, pp. 590-601, May 2006.

[5] M. Sonka, X. Zhang, M. Siebes, M. S. Bissing, S. C. DeJong, S. M. Collins, C. R. Mckay, "Segmentation of Intravascular Ultrasound Images: A Knowledge-Based Approach,” IEEE Tran. Med. Imag., vol. 14, no. 4, pp. 719-732, Dec. 1995.

[6] Gozde B. Unal, S. Bucher, S. Carlier, Gregory G. Slabaugh, Tong Fang, K. Tanaka: Shape-Driven Segmentation of the Arterial Wall in Intravascular Ultrasound Images. IEEE Tran. Info. Tech. Biomed., vol. 12, no. 3, pp. 335-347, 2008.

[7] J. D. Klingensmith, R. Shekhar, D. G. Vince, "Evaluation of Three-Dimensional Segmentation Algorithms for the Identification of Luminal and Medial-Adventitia Borders in Intravascular Ultrasound Images,” IEEE. Tran. Med. Imag., vol. 19, no. 10, 2000.

[8] J. Besag, “On the Statistical Analysis of Dirty Pictures”, Journal of Royal Statistical Society, Series B, vol. 48, no. 3, pp. 259-302, 1986.

[9] F. Meyer and R. R. Coifman, "Brushlets: A tool for directional image analysis and image compression,” Applied and computational harmonic analysis, vol. 4, pp. 147-187, 1997.

[10] P. Ausher, G. Weiss, M. V. Wickerhauser, "Local sine and cosine bases of Coifman and Meyer and the construction of smooth wavelets," in Wavelets- A tutorial in Theory and Application, vol. 2, Wavelet Analysis and its Applications, C. K. Chui, Ed. San Diego: Academic Press, 1992, pp. 237-256.

[11] D. Mumford, J. Shah, "Optimal Approximation by Piecewise Smooth Functions and Associated Variational Problems," Communication on Pure and Applied Math. Vol. 42, pp. 544-685. 